\title{
SEGURANÇA DO AMBIENTE USANDO DISPOSITIVO IOT COM PROCESSAMENTO DISTRIBUÍDO
}

\author{
Bruno J. G. Praciano, Francisco Lopes de Caldas Filho, Lucas M. C. e Martins, \\ Dayanne F. da Cunha, Daniel Alves da Silva e Rafael Timóteo de Sousa Júnior \\ Universidade de Brasília - Departamento de Engenharia Elétrica, Brasília - DF, Brasil - Zipcode 70910-900
}

\begin{abstract}
RESUMO
Nos últimos tempos, o conceito de reconhecimento facial ganhou grande popularidade. Graças à evolução da Internet das coisas (IoT), as ideias para monitorar com smartphones e outros dispositivos agora parecem possíveis. Problemas como o reconhecimento facial para entrada em ambientes estão sendo abordados pela IoT. Neste artigo, apresentamos um sistema integrado de dispositivos inteligentes Fog baseado em IoT. A solução proposta de uma implantação no local de um módulo de IoT que é usado para monitorar e anunciar a pessoa que entrou em uma sala, por exemplo. E o aplicativo bot também é fornecido, permitindo que o usuário final verifique os rostos de acordo. Esse artigo também descreve uma visão de alto nível da arquitetura do sistema. No final, é discutido o funcionamento do sistema na forma de um caso de uso que comprova a correção do modelo proposto.
\end{abstract}

\section{PALAVRAS-CHAVE}

IoT, Reconhecimento Facial, Machine Learning, Fog

\section{INTRODUÇÃO}

Como grande parte da população vive em áreas urbanas e tem acesso à Internet, a quantidade de dados gerados está aumentando, portanto, é necessário adequar-se a essa nova realidade [Gubbi et al.]. Uma das razões para esse aumento é que as pessoas estão usando dispositivos IoT diariamente, como telefones celulares, TV inteligente, relógios inteligentes e veículos conectados; consequentemente, é necessário usar técnicas de análise de dados para entender o comportamento dessa grande quantidade de informações [Rathore et al.].

Com os recentes avanços tecnológicos, novas soluções para coletar informações ambientais e executar automaticamente tarefas no mundo "real" foram adotadas de uma maneira anteriormente inimaginável. A Internet das Coisas (IoT) surgiu propondo um gerenciamento automatizado da cadeia de suprimentos viável e passou a ser usada em outros campos, como assistência médica, automação residencial e cidades inteligentes [Shi \& Dustdar].

A tecnologia IoT está crescendo em muitas áreas de pesquisa e isso é causado por sua capacidade de coletar e enviar dados com o mínimo de interferência humana. Os dados são coletados pelos sensores ou câmeras presentes nos dispositivos e podem ser usados em aplicações estratégicas, como monitoramento climático ou monitoração interna de edifícios, para melhorar o gerenciamento, como reconhecimento facial [Swamy \& Sowmyarani].

Mas esse tipo de software precisa reunir muitos dados para ser mais eficiente e assertivo. Lidar com esse grande volume de dados é um desafio em todas as áreas de tecnologias. O uso da inteligência artificial é comum para aprimorar uma nova análise e processo de construção de novos dispositivos [Tsai et al.].

Recentemente, adotamos o conceito de Fog Computing para conectar aplicativos em muitos dispositivos inteligentes. A ideia é criar uma camada entre os gadgets e a nuvem, alocando os recursos próprios da organização para serem usados adequadamente. Esse paradigma foi desenvolvido para aplicações em tempo real, como abordagens de big data e ciência de dados, e com isso é possível aumentar a velocidade de transmissão e diminuir a latência [Stojmenovic]. 
As técnicas de detecção de faces se tornaram comuns após o algoritmo de detecção de objetos Viola-Jones [Viola \& Jones]. Essa estrutura pode ser treinada para detectar muitos tipos de classes de objetos; no entanto, foi construída para resolver o problema de detecção de face. O reconhecimento disso deve-se permitir que a câmera detecte rostos em tempo real usando hardware de baixo custo e é necessário enfatizar que este procedimento forneceu uma baixa porcentagem de falsos positivos.

Atualmente, existem outras técnicas com mais eficiência para realizar essa tarefa em nosso trabalho mostramos um breve resumo das bibliotecas de código-fonte aberto que utiliza aprendizado de máquina, sendo: Face Recognition [Geitgey \& Nazario], OpenFace [Amos \& Satyanarayanan] e Dlib [King], que nos ajudam a modelar o ambiente IoT de detecção de faces usando Fog Computing.

O objetivo deste trabalho é construir um dispositivo inteligente usando computação Fog e coordenar seu reconhecimento de rosto remotamente. Os benefícios do uso dessa arquitetura são de baixo custo para implementar e operar e a possibilidade de trabalhar com nuvens federadas.

Além desta introdução, este artigo está organizado da seguinte forma: na Seção II, apresentamos os trabalhos relacionados. Na Seção III, apresentamos o Middleware IoT proposto para reconhecimento de face usando o Fog Computing. Na Seção IV, apresentamos a metodologia de teste e os resultados. Finalmente, na Seção V, apresentamos conclusões e sugestões gerais para trabalhos futuros.

\section{TRABALHOS RELACIONADOS}

A IoT e o reconhecimento facial foram tópicos importantes na pesquisa acadêmica nos últimos anos e a combinação desses tópicos pode ajudar em vários problemas atuais, como encontrar pessoas perdidas usando câmeras de vigilância.

O trabalho de [Amin et al.] descreve um esquema de reconhecimento de rosto descentralizado usando câmeras de vigilância distribuídas usando IoT em conjunto com arquitetura em nuvem. Eles usaram o método Viola-Jones [Viola \& Jones]. Implementado através da biblioteca de visão computacional OpenCV como o método oficial de detecção de rosto, chamado Haar Cascades. O foco deste estudo é apresentar o processo de extração de faces em um dispositivo local e enviá-lo para que a classificação seja realizada em nuvem.

Em [Mano et al.] é apresentado outro estudo sobre este assunto, que utiliza IoT para melhorar os hospitais usando conceitos de dispositivos inteligentes, usando as características faciais dos pacientes, é possível identificá-lo e também reconhecer a emoção do paciente. Capturar a foto e processar a detecção e o reconhecimento de faces em um dispositivo na rede local do paciente e, em seguida, envia apenas as informações relevantes para as camadas superiores a serem mostradas no sistema. Foram utilizadas técnicas como k-Nearest Neighbor (kNN) e Support Vector Machine (SVM) [Mitchell] para identificar o paciente e o Face Tracker Algorithm [Libralon \& Romero] para detectar rostos usando 66 pontos de recurso.

[Hossain et al.] apresentaram um trabalho que usa a IoT e a geometria da face para fazer a identificação baseada em biometria facial para facilitar a autenticação. Neste artigo, os autores mostram uma solução modelada que inclui sensores locais que enviam dados para dispositivos de comunicação em nuvem. Os dispositivos de comunicação são aqueles que atuam como gateways de IoT.

\section{MIDDLEWARE IOT PROPOSTO PARA RECONHECIMENTO DE FACE USANDO O FOG COMPUTING}

O objetivo deste artigo é desenvolver uma solução de identificação por meio do reconhecimento de face usando processamento distribuído, reduzindo assim a necessidade de enviar dados de streaming para a nuvem e aumentar a flexibilidade de mover o hardware para o processamento local. À medida que a qualidade da câmera aumentou, houve um crescimento exponencial na quantidade de soluções de segurança de dados necessárias para transmitir. A arquitetura proposta tem como objetivo armazenar e processar dados localmente, relatando eventos de interesse do usuário. 


\subsection{Processo Proposto}

O processo monitoramento é realizado em três etapas: registro, treinamento e monitoramento.

A etapa de registro é a primeira e consiste na inclusão de imagens de pessoas feitas por um usuário. Este usuário deve registrar pessoas, sendo necessário informar o nome e algumas fotos de seus rostos. Para melhorar a precisão do reconhecimento, é importante que pelo menos três fotos de cada pessoa sejam registradas.

A etapa de treinamento ocorre quando as pessoas e suas fotos são submetidas a um algoritmo de aprendizado de máquina para gerar um modelo que será utilizado para realizar a identificação facial e reconhecimento da pessoa cadastrada previamente nessa base de dados.

Finalmente, na etapa de monitoramento as câmeras de vídeo são usadas para monitorar uma área para identificar pessoas conhecidas. Nesse passo, dois mecanismos são usados continuamente: detecção de rosto para detectar a presença de um rosto em uma imagem e, ao detectar uma pessoa, reconhecimento de rosto. Em seguida, conforme configurado anteriormente, são gerados alertas e notificações sobre as pessoas detectadas nas imagens.

\subsection{Arquitetura Proposta}

A solução é baseada no uso dos paradigmas IoT, Fog e Cloud Computing. Ao reconhecer um usuário, o dispositivo envia uma notificação ao Middleware informando que um usuário específico entrou na sala, permitindo que sensores e dispositivos inteligentes sejam controlados de acordo com o perfil. O Fog Computing oferece a possibilidade de usar recursos distribuídos na rede interna ou em sua borda. A computação em nuvem oferece a possibilidade de usar recursos de computação praticamente infinitos.

A solução proposta envolve câmeras de vídeo, um dispositivo inteligente, um sistema de registro e um módulo de treinamento, conforme mostrado na Figura 1.

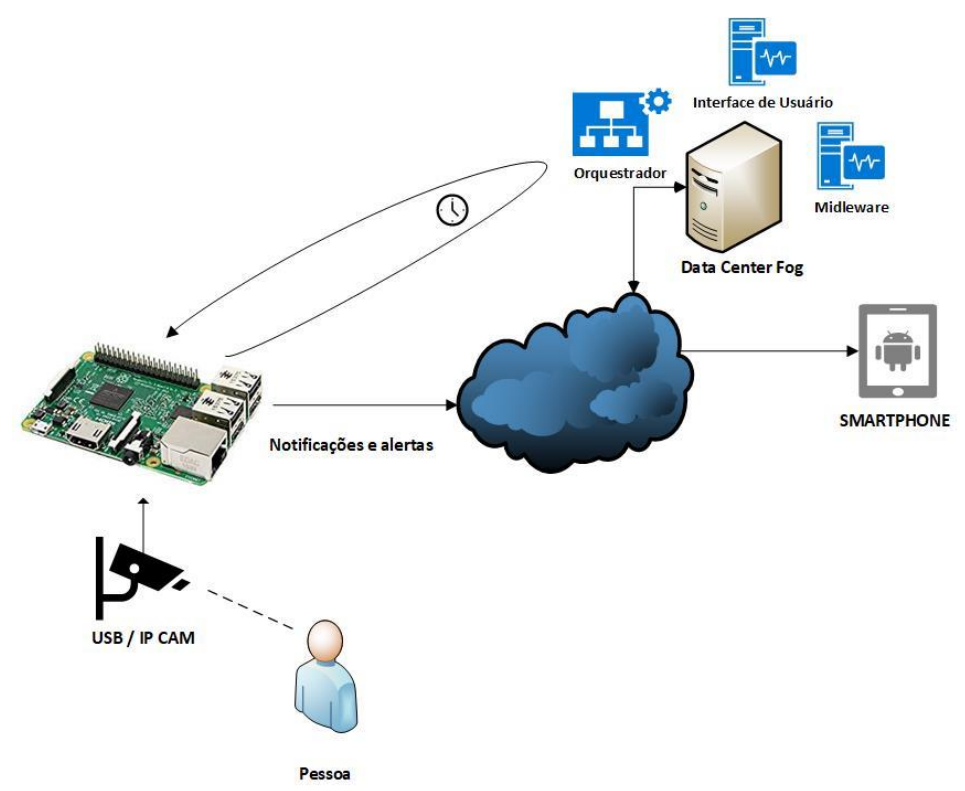

Figura 1. Arquitetura de solução de câmera inteligente usando Fog Computing

1) Interface do usuário (UI): A interface do usuário (UI) é o ambiente no qual o usuário pode interagir com a funcionalidade de registro do sistema. Esse componente é utilizado em nuvem. O registro das pessoas ocorrerá no UIMS [Dutra et al.]. O UIMS enviará ao orquestrador as imagens a serem usadas no treinamento do algoritmo.

2) Orquestrador: o orquestrador é instalado utilizando arquitetura de Fog computing perfeitamente com outros dispositivos. Ele tira proveito do recurso de composição de $F o g$, como alocação de recursos e proximidade do usuário. 
3) Módulo de Treinamento utilizando Fog: o módulo de treinamento pode ser instalado em um datacenter em nuvem remoto ou em um datacenter de rede local. Sua responsabilidade é receber o registro e o treinamento do algoritmo que será enviado aos dispositivos inteligentes. Esse transformará as imagens em casamentos de 128 dimensões, gerando um arquivo pickle que salvará todas as informações no formato de bytes. Este arquivo contém informações sobre cada foto no formato de incorporação, garantindo que cada imagem seja exclusiva no conjunto de dados gerado anteriormente.

4) Câmeras de vídeo: As câmeras de vídeo devem estar operando no ambiente físico a ser monitorado, conectado ao SmartDevice por conexão USB ou LAN. É importante ressaltar que as câmeras IP podem ser instaladas na rede local ou na Internet, mas sua operação será dificultada pela maior latência inerente a esse ambiente.

5) Dispositivo inteligente: o dispositivo inteligente instalado na rede local interage com as câmeras via interface USB ou interface IP, desde que seja possível acessar o streaming de vídeo através de qualquer uma dessas interfaces.

O dispositivo inteligente consiste em módulos de deteç̧ão, identificação, sincronização e notificação. Existem dois dispositivos físicos locais para processamento distribuído, um para detecção de face e outro para classificação de face. Quando faces conhecidas são encontradas, elas são enviadas para um módulo de sincronização na nuvem para possíveis alertas.

O fluxo da imagem é submetido a um método de detecção de rosto chamado Histogramas de Gradientes Orientados (HOG) [Dalal \& Triggs], que detecta a presença de rostos em um quadro. Quando um rosto é detectado em uma imagem, ele é destacado e enviado ao módulo de identificação via soquete TCP. Mais de uma face pode ser identificada ao mesmo tempo, encontrando cenários com mais de uma pessoa na porta por vez.

As imagens dos rostos identificados, serão convertidas em 68 pontos para o alinhamento do rosto a partir do algoritmo chamado estimativa do marco da face [Kazemi \& Sullivan]. Logo após esse alinhamento, é gerada uma incorporação 128-D compacta, usando um modelo pré-treinado, baseado na função de perda baseada em tripletos, a partir do método apresentado em [Schroff \& Philbin]. O modelo foi pré-treinado e disponibilizado pelo projeto OpenFace [Amos \& Satyanarayanan]. Após gerar esses pontos, usamos um classificador chamado SVM (Support Vector Machine) [Rüping] para comparar os 128 componentes de face encontrados com os rostos registrados em seu banco de dados. Esta análise pode resultar em identificação do usuário ou informar que não existe esse rosto cadastrado na base de dados.

O módulo de notificação envia ao IoT Middleware que alguém entrou na sala. Será enviado junto com a mensagem, a foto capturada pela câmera e o nome da pessoa reconhecida. Se a pessoa não tiver sido identificada, o nome desconhecido será enviado. Também é enviada uma notificação ao programa de mensagens instantâneas para facilitar a visualização do evento pelo usuário.

O módulo de sincronização é responsável pela comunicação com o nó Fog e a nuvem para receber atualizações do banco de dados e enviar telemetria de integridade do dispositivo para esse controlador. Esse processo é descrito em mais detalhes na Seção 3.3.

\subsection{Processamento dos Dados e Sincronização}

O treinamento de modelos de aprendizado de máquina e análise de vídeo são atividades que exigem muitos recursos computacionais de processamento, armazenamento e largura de banda da rede. Com o objetivo de simplificar e diminuir os custos da solução, optamos por aproveitar os recursos dos paradigmas de IoT e Fog Computing, e os principais recursos explorados em nossa solução foram a distribuição de recursos e a cooperação de dispositivos.

A distribuição de recursos ocorre porque as atividades que exigem mais recursos computacionais são executadas em computadores remotos, baseados em nevoeiro ou na nuvem. $\mathrm{O}$ resultado desse processamento é propagado para recursos com menos poder de processamento, como o Smart Device.

O Fog Trainer busca dados do banco de dados de middleware da IoT e realiza o treinamento. Esse novo treinamento é enviado para as câmeras inteligentes que fazem parte da rede. A Smart Câmera envia a telemetria de sua operação ao Fog Trainer para apoiar sua decisão sobre o treinamento a ser realizado. 
Por fim, o dispositivo remoto irá fazer consultas periódicas ao controlador Fog, verificando uma nova versão do algoritmo treinado para download. Depois de obter o algoritmo treinado, o dispositivo inteligente transmitirá dados das câmeras locais para iniciar o processo de reconhecimento de rosto.

\section{TESTES E RESULTADOS}

Esse protótipo foi instalado na porta de acesso do nosso laboratório, em um ângulo em que era possível detectar a entrada de pessoas pela porta.

A interface do usuário foi instalada na nuvem. O Trainer Fog e o Orquestador foram instalados nos computadores da rede local. O Smart Device foi construído usando um Raspberry Pi III e foi conectado a uma câmera conectada à interface USB com resolução Full HD e uma taxa de atualização de 11 quadros por segundo. O módulo de notificação foi configurado para enviar as informações ao middleware e a um bot do Telegram.

Para esse experimento, 11 pessoas diferentes foram registradas no sistema, entre um universo de 30 pessoas que participavam do laboratório. Conforme destacado na Figura 2, foram usadas fotos dos rostos tirados da própria câmera, bem como fotos capturadas utilizando smartphones.

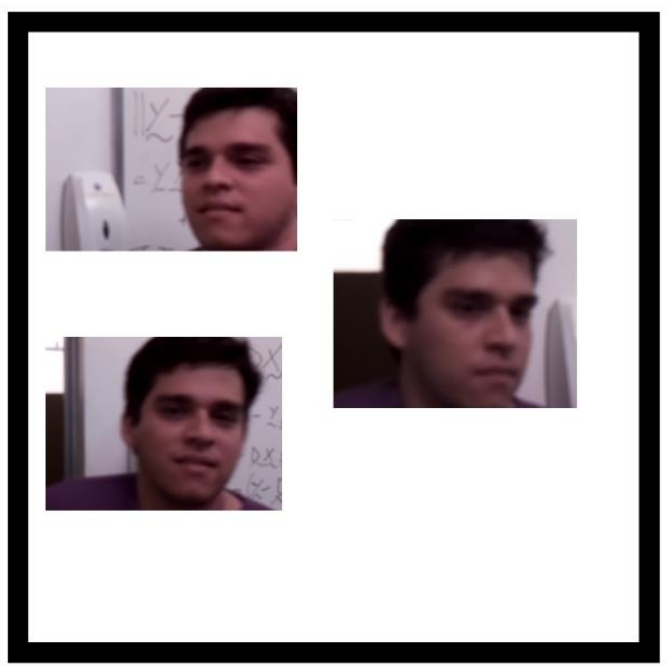

(a) Dataset com images de treinamento do Bruno

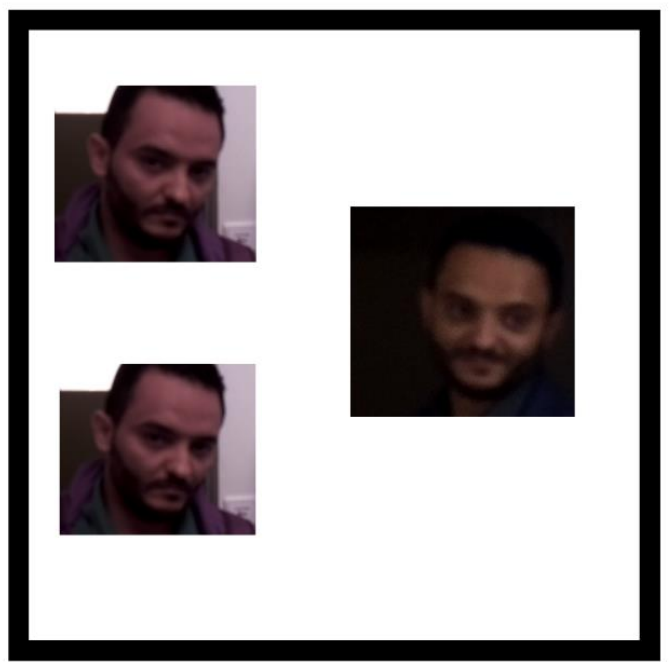

(b) Dataset com images de treinamento do Francisco

Figura 2. Exemplo de conjunto de treinamento para dois usuários conhecidos

Durante 48 dias de experimentos, 802 imagens de rostos foram identificadas em condições de luz ambiente que variavam de acordo com os diferentes períodos do dia. Vinte e cinco por cento de todas as imagens obtidas foram de pessoas registradas no sistema de reconhecimento facial, e o algoritmo teve êxito para sua classificação de forma correta em $80,40 \%$ dos casos, como mostrado na Figura 3. A solução proposta falhou em identificar 27,03\% das imagens classificadas como desconhecidas e na Figura 4 descreve essa abordagem, pois foram incluídas no banco de dados. A classificação ocorreu aproximadamente em $75 \%$ de todas as amostras obtidas. 


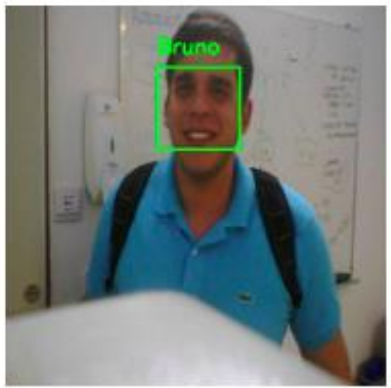

(a) Bruno

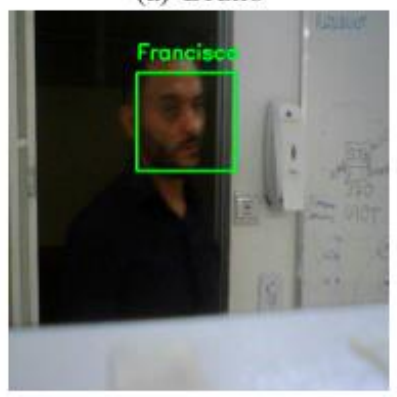

(c) Francisco

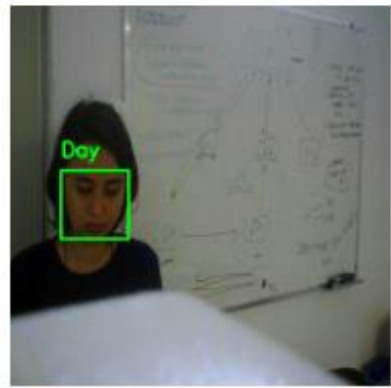

(b) Day

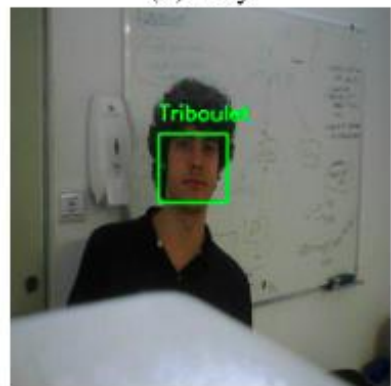

(d) Tribouillet

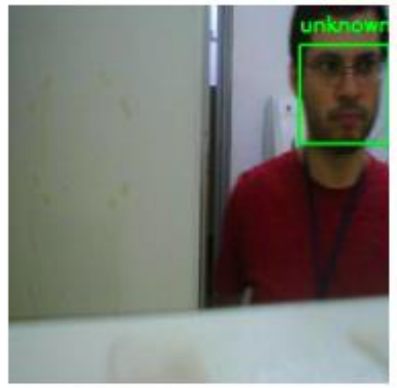

(e) Unknown

Figura 3. Reconhecimento correto dos membros do laboratório através da solução proposta

\section{CONCLUSÕES E TRABALHOS FUTUROS}

Neste trabalho, desenvolvemos uma solução menor, mais leve e com menor consumo de energia. Os resultados de nossa solução proposta são altos, e o desenvolvimento do modelo foi possível alcançar esses números devido ao treinamento ter sido realizado de forma distribuída. De acordo com nossos resultados, uma precisão próxima a $80 \%$ é obtida para pessoas conhecidas e $72,96 \%$ para pessoas desconhecidas.

Para trabalhos futuros, esperamos incluir uma quantidade maior de faces em nosso conjunto de dados para aumentar os resultados. Também pretendemos usar algoritmos diferentes combinados com duas câmeras que podem ser implementadas para obter um desempenho superior. 


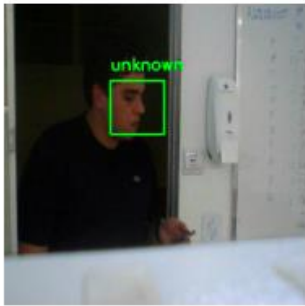

(a) Bruno not recognized.

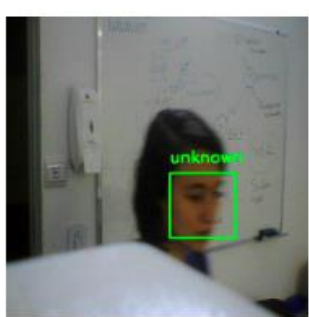

(c) Day not recognized.

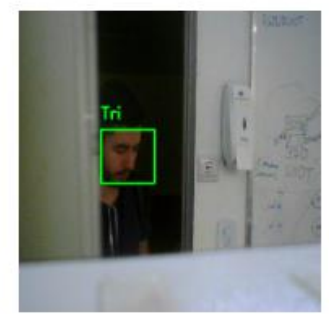

(b) Cassio identified as Tribouillet.

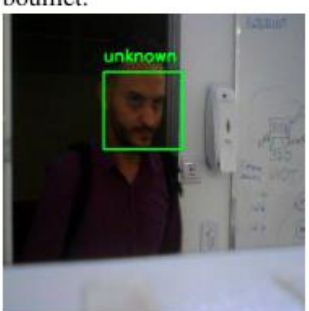

(d) Francisco not recognized.

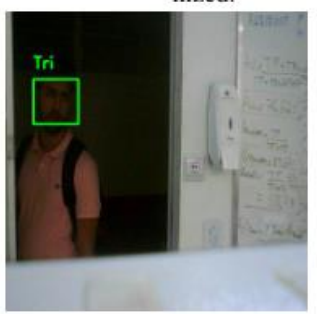

(e) Rafael identified as Tribouillet.

Figura 4. Identificação incorreta de membros do laboratório através de solução proposta

\section{AGRADECIMENTO}

Os autores agradecem o apoio financeiro dos Conselhos de Pesquisa do Brasil CNPq (Convênio 465741 / 2014-2 INCT sobre segurança cibernética), CAPES (Convênio 23038.007604 / 2014-69 FORTE), FAP-DF (Bolsas 0193.001366 / 2016 UIoT e 0193.001365 / 2016 SSDDC), bem como o Laboratório LATITUDE / UnB (Convênio 23106.099441 / 2016-43 SDN), o Ministério da Economia (Convênio 005/2016 DIPLA e 083/2016 ENAP) e o Escritório de Segurança Institucional da Presidência da República da Brasil (Convênio 002/2017).

\section{REFERÊNCIAS}

Amin, A. H. M., Ahmad, N. M., \& Ali, A. M. M. (2016, Maio). Decentralized face recognition scheme for distributed video surveillance in IoT-cloud infrastructure. In 2016 IEEE Region 10 Symposium (TENSYMP) (pp. 119-124). IEEE.

Amos, B., Ludwiczuk, B., \& Satyanarayanan, M. (2016). Openface: A general-purpose face recognition library with mobile applications. CMU School of Computer Science, 6.

Dalal, N., \& Triggs, B. (2005, Junho). Histograms of oriented gradients for human detection.

Dutra, B. V.; Alencastro, J. F.; Caldas Filho, F. L.; Martins, L. M. C. e; de Sousa Júnior, R. T.; Albuquerque, R. de O. (2019, Junho). HIDS by signature for embedded devices in IoT networks. In Actas de las V Jornadas Nacionales de Ciberseguridad (JNIC 2019) (pp. 53-61). Universidad de Extremadura.

Geitgey, A. \& Nazario, J. (2017), Repositório de código [Face Recognition]. Consultado em 2019, Julho 30. Disponível em https://github.com/ageitgey/face_recognition/ 
Hossain, M. S., Muhammad, G., Rahman, S. M. M., Abdul, W., Alelaiwi, A., \& Alamri, A. (2016). Toward end-to-end biometrics-based security for IoT infrastructure. IEEE Wireless Communications, 23(5), 44-51.

Jin, J., Gubbi, J., Marusic, S., \& Palaniswami, M. (2014). An information framework for creating a smart city through internet of things. IEEE Internet of Things journal, 1(2), 112-121.

Kazemi, V., \& Sullivan, J. (2014). One millisecond face alignment with an ensemble of regression trees. In Proceedings of the IEEE conference on computer vision and pattern recognition (pp. 1867-1874).

King, D. E. (2009). Dlib-ml: A machine learning toolkit. Journal of Machine Learning Research, 10(Jul), 1755-1758.

Libralon, G. L., \& Romero, R. A. F. (2014, Outubro). Mapping of facial elements for emotion analysis. In 2014 Brazilian Conference on Intelligent Systems (pp. 222-227). IEEE.

Mano, L. Y., Faiçal, B. S., Nakamura, L. H., Gomes, P. H., Libralon, G. L., Meneguete, R. I., ... \& Ueyama, J. (2016). Exploiting IoT technologies for enhancing Health Smart Homes through patient identification and emotion recognition. Computer Communications, 89, 178-190.

Mitchell, T. M., \& Learning, M. (1997). Mcgraw-hill science. Engineering/Math, 1, 27.

Rathore, M. M., Ahmad, A., \& Paul, A. (2016, Outubro). IoT-based smart city development using big data analytical approach. In 2016 IEEE international conference on automatica (ICA-ACCA) (pp. 1-8). IEEE.

Rüping, S. (2001). SVM kernels for time series analysis (No. 2001, 43). Technical Report, SFB 475: Komplexitätsreduktion in Multivariaten Datenstrukturen, Universität Dortmund.

Schroff, F., Kalenichenko, D., \& Philbin, J. (2015). Facenet: A unified embedding for face recognition and clustering. In Proceedings of the IEEE conference on computer vision and pattern recognition (pp. 815-823).

Shi, W., \& Dustdar, S. (2016). The promise of edge computing. Computer, 49(5), 78-81.

Stojmenovic, I. (2014, Novembro). Fog computing: A cloud to the ground support for smart things and machine-to-machine networks. In 2014 Australasian Telecommunication Networks and Applications Conference (ATNAC) (pp. 117-122). IEEE.

Swamy, S. N., \& Sowmyarani, C. N. (2018, Março). Repeated data management framework for IoT: A case study on weather monitoring and forecasting. In 2018 4th International Conference on Recent Advances in Information Technology (RAIT) (pp. 1-7). IEEE.

Tsai, C. W., Lai, C. F., Chiang, M. C., \& Yang, L. T. (2013). Data mining for internet of things: A survey. IEEE Communications Surveys \& Tutorials, 16(1), 77-97.

Viola, P., \& Jones, M. (2001). Rapid object detection using a boosted cascade of simple features. CVPR (1), 1(511-518), 3. 